Carvalho, Agda.

Universidad Anhembi Morumbi- PPG Design. UNESP- Instituto de Artes.

Trovo, Priscila.

Universidad Anhembi Morumbi- PPG Design. Fiam Faam Centro Universitario.

Kishimoto, Grace.

Universidad Anhembi Morumbi- PPG Design. Universidad Presbiteriana Mackenzie.

Rosa, Natalia.

Universidad Anhembi Morumbi- PPG Design.

Kulaif, Patricia.

Universidad Anhembi Morumbi- PPG Design.

Valli, Adriana.

Universidad Anhembi Morumbi- PPG Design. Fiam Faam Centro Universitario.

Braga, Rechilene Maia.

Universidad Anhembi Morumbi- PPG Design.

Da Hora, Miguel.

Universidad Anhembi Morumbi- PPG Design.

\title{
Proyecto interdisciplinar Feeling the senses: El vestir como experiencia.
}

\section{Interdisciplinary project Feeling the senses: Dressing as an experience.}

TIPO DE TRABAJO:

Comunicación Virtual.

PALABRAS CLAVE:

Interdisciplinariedad; cotidiano; vestir; corsé.

KEY WORDS:

Interdisciplinarity; daily life; dressing; corset.

\section{RESUMEN.}

El texto introduce un debate sobre el cuerpo y el vestir al dialogar con las incertidumbres y las complejidades de la vida cotidiana plural, centrándose en las inquietudes y las subjetividades corpóreas del movimiento en el espacio. Se destaca esta experiencia con la estructura que elabora el vestir, en el instante en que se evidencia la visceralidad del cuerpo, durante la percepción de los aspectos sensitivo-cognitivos.

Entre las referencias se observa el corsé, una pieza del vestuario femenino del siglo XIX, cuya estructura interfería en la postura y el movimiento, modelando la silueta y requiriendo una organización corporal, que se vinculaba a las reglas sociales y culturales de comportamiento. Este proyecto interpela a la experiencia del cuerpo femenino con el vestir y las imposiciones actuales, que están camufladas o son nebulosas, pero que interfieren en la experiencia del cuerpo. De este interrogante surge 
el vestible denominado Feeling the Senses, investigación que comenzó en 2016, como una propuesta que articula, tanto en el proceso como en la realización, los campos del arte, el diseño y la tecnología. Del debate y la producción surge un equipo multidisciplinario de investigadores, que forman el grupo de investigación diseño y cuerpo: aspectos del proyecto en el arte y la moda, coordinado por Agda Carvalho.

Con una aproximación que se centra en la no linealidad de los comportamientos y en la inestabilidad de los espacios, la estructura busca soluciones que mezclen la rigidez y la flexibilidad del entorno. La materialidad y el diseño estimulan un aspecto rígido, pero los movimientos corpóreos exponen la situación inestable que rodea lo cotidiano del cuerpo y del vestir. Esta estructura también interfiere en la postura, el movimiento y la organización corporales, modelando en la silueta femenina las reglas sociales, respondiendo a las continuas modificaciones y reorganizaciones contemporáneas.

\section{ABSTRACT.}

The text provides a discussion of body and dress, to interact with the uncertainties and complexities of a multiple daily life, which is focused on tangible concerns and subjectivities in space. We highlight this experience with the structure that draws the dressing, at the moment when the viscerality of the body is evident, during the perception of the sensory-cognitive aspects.

Among the references, the corset, a piece of nineteenth-century women's clothing, whose structure interfered with posture and movement, modeling the silhouette and requesting a corporal organization, which was linked to social and cultural rules of behavior. This project challenges the experience of the female body with the dress, the impositions and current norms, which are often camouflaged or nebulous, but interfered in the experience of the body. This question arises Wearable called Feeling the Senses, research started in 2016, as a proposition which articulates both the process and the implementation, the fields of art, design and technology. Discussion and production comes an interdisciplinary team of researchers, who take part in the research group design and body: projective aspects in art and fashion, coordinated by Agda Carvalho.

With an approach focused on the non-linearity of behaviors and the instability of spaces, the structure seeks solutions that blend the rigidity and flexibility of the environment. The materiality and the design simulate a hard aspect, but the corporeal movements expose the unstable situation that surrounds the daily life of the body and the dress. This structure also interferes with posture, movement and body organization, modeling on thebody sillhoutte the social rules, responding to continuous modifications and contemporary reorganizations.

\section{CONTENIDO}

\section{Introducción.}

El texto trata la articulación del cuerpo y del vestirse como experiencia, expone el sentido de la subjetividad corpórea cuando entra en coalición con las diversidades del sentir y del vivir. En este proceso revela la incertidumbre y las complejidades de un cotidiano plural, al abordar los aspectos sensoriales y cognitivos que transbordan con el movimiento espacial del cuerpo, así como discute las articulaciones con las materialidades. Con este proyecto interdisciplinario se destaca un recorrido colaborativo, expuesto por medio del entrelazado de aspectos conceptuales y del proceso de realización de la pieza vestible Feeling the senses.

Este proyecto empezó en 2016, con un equipo interdisciplinar ${ }^{1}$ de investigadores que actúan en el campo del arte, del diseño y de la tecnología. La investigación parte de la idea del corsé, una pieza importante de la historia de la moda femenina, que surgió en el siglo XVI cuando las mujeres de la aristocracia española e italiana empezaron a usar un tipo de sujetador estructurado con aletas de ballena, con el objetivo de crear una postura rígida en el cuerpo del usuario. El enfoque referencial de este proyecto está específicamente en el uso de esta pieza en el siglo XIX, un período con reglas sociales y normas de comportamiento, un momento en el que el corsé integra el vestuario y contribuye con las normas de sociabilidad.

A partir de estas investigaciones surge el proyecto interdisciplinario que dirige las discusiones de los integrantes para la realización de un objeto articulado con la inconstancia y la inestabilidad social en lo contemporáneo. Por medio de experimentos de estructuras acopladas al cuerpo, se desarrollan las discusiones y la materialización de las propuestas que discuten la adaptabilidad y las cuestiones íntimas y subjetivas de la relación cuerpo y vestuario.

\footnotetext{
${ }^{1}$ Grupo de investigación Cnpq: Diseño y cuerpo: aspectos proyectuales en el arte y la moda liderado por la Prof. Dra. Agda Carvalho. Investigadores: Priscila Trovo, Grace Kishimoto, Natalia Rosa, Patrícia Kulaif, Adriana Valli, Rechilene Maia Braga, Miguel da Hora, Marcelo C. Oliveira, Edilson Ferri y Nivia Ferreira.
} 
En este proceso se observa que las imposiciones y las normas actuales en muchos momentos están camufladas o nebulosas, pero aún así interfieren en la realización de estructuras, que no se circunscriben a una condición rígida del comportamiento, ya que se da una involucración de la experiencia del cuerpo y del vestirse con el cotidiano plural.

Esta inestabilidad de los espacios señala un recorrido sesgo, que trata la mezcla de los estímulos y los procesos en que los distintos aspectos que nos circundan instigan la potencia del cuerpo por medio del proyecto de diseño, que está continuamente reorganizándose con los experimentos y las discusiones del equipo interdisciplinario.

\section{Cotidiano/ Vestirse.}

Las características de un cotidiano plural se evidencian en la red de posibilidades de un sistema de complejidades, en el que un entretejido de eventos, acciones e interacciones que constituyen el mundo fenoménico se presentan como un conjunto desordenado e incierto. Según Morin (2007), a primera vista, la complejidad es un fenómeno cuantitativo, es decir, entendido como una cantidad de interacciones e interferencias entre determinado número de agentes en un sistema. Sin embargo, no comprende solamente la cantidad de agentes y sus interacciones, sino también la incertidumbre y las indeterminaciones de los fenómenos aleatorios que ocurren cuando suceden estas interacciones.

Sujetos y objetos se relacionan de forma mutua, en la cual el sujeto piensa, define y observa y el objeto se permite reconocer, definir y pensar. De esta forma, no hay objeto que no esté relacionado a un sujeto y no hay sujeto que no se relacione a un objeto. Así, el objeto y el sujeto sucediendo separadamente no son suficientes (MORIN, 2007).

El sujeto emerge al mismo tiempo que el mundo, desde un punto de partida sistémico, sobre todo a partir de su auto-organización, autonomía e individualidad. Así, para Morin (2007), el sujeto y el objeto aparecen como emergencias inseparables de la autoorganización del sistema del mundo, por lo que son indisociables. Tanto sujeto como objeto deben permanecer abiertos para influenciar y dejarse influenciar el uno por el otro.

Es en esta red de articulaciones que las estructuras que componen el vestirse interfieren en las respuestas corpóreas y en los aspectos sensoriales y cognitivos del usuario, y en esta situación despiertan las manifestaciones subjetivas y viscerales. Se destaca que nos confrontamos de forma diversa con la experiencia, ya que cada cuerpo está conectado con las situaciones de un contexto y con los micro mundos que determinan el comportamiento y la actitud, que está expuesta con la organización corpórea (VARELA,2003). Esta miscelánea de elementos lleva al cuerpo a una ebullición de sentimientos y de sensaciones con la pieza vestible Feeling the senses.

Pero esta subjetividad global del cuerpo es ella misma la reunión de una multiplicidad de subjetividades solidarias unas con otras, según estructuras de jerarquización al mismo tiempo complejas y en constante mutación, pues la armonía, aquí como en otros lugares, no procedería apenas de una lucha. (LINS, 2002, p. 77)

La experiencia corpórea con lo vestible expone el sistema complejo cotidiano, que está camuflado con algunas reglas y con una cierta flexibilidad, o sea, las normas sociales pueden adaptarse y modificarse en la contemporaneidad. La reflexión de la relación de las reglas de comportamiento sociales y el cuerpo femenino está referenciada en la rigidez del corsé, en el siglo XIX, porque es el instante en que el cuerpo entabla un embate con el vestuario y gradualmente dirige a la mujer en la búsqueda de una silueta. Es importante destacar que, a principios del siglo XIX, el cuerpo femenino usa un vestuario libre y sin ataduras en la estructura, pero aún está preso a las reglas sociales de comportamiento. El proyecto de la pieza vestible Felling the Senses está basado en esta pieza del vestuario femenino, que en su primera forma de corsé era llamada de sujetador, en español "corpiño", en italiano "corpetto", en francés "corps à la baleine", en inglés "whalebone bodies". Es relevante destacar el hecho de que este primer nombre deriva de la palabra "cuerpo", ya que evidencia la relación simbiótica de la vestimenta y del cuerpo femenino. El corsé, al mismo tiempo que moldea el cuerpo, también se ve moldeado por él. Además de ser importante para la historia de la moda, el corsé también es una pieza fundamental en la historia del cuerpo femenino. Desde el siglo XVI hasta hoy, el corsé ha tomado varias formas: desde los sujetadores con aletas de ballena, pasando por las fajas enrigidecidas por placas de madera, los corsés saludables y flexibles, y su reencarnación en el siglo XX como cintas elásticas (STEELE, 2011). Éstas modelan el cuerpo, pero permiten movimientos dentro de un límite, que se determina con la resistencia del material y la capacidad de expansión.

En el Proyecto Felling the Senses las percepciones del vestirse posibilitan otros descubrimientos, evidencian una vivencia múltiple del vestirse y la apertura de mundos. El organismo cuerpo adquiere otras formas en el espacio, ya que la estructura reacciona a los movimientos físicos, a las tensiones y las subjetividades. Lo vestible posee en su esencia, entre otros aspectos, una deconstrucción de lo esperado, éste presenta para el cuerpo otras maneras de enfrentamiento del contexto, diferentes de la estructura rígida del corsé.

La diversidad de operaciones que actúan simultáneamente entre los agentes estimulan la negociación durante el acontecimiento perceptivo, esta condición lleva a la interferencia, a la modificación corpórea y a la emergencia de atributos sensoriales y cognitivos 
(VARELA, 2003). De la multiplicidad de conexiones y micro procesos cotidianos irrumpen la intimidad del cuerpo del usuario y se exponen las tonalidades del sentir y del vivir.

Cualquier estudio sobre la experiencia humana es, por necesidad, multifacético (MORIN, 2007), porque este cuerpo está inmerso en un contexto de tradiciones sociales y éticas, más allá de un entendimiento como cuerpo biológico. La experiencia corpórea se potencia en la relación con lo vestible que reacciona a su entorno cultural, porque sus acciones y movimientos se dan a partir de la manifestación, desde adentro hacia afuera, de las subjetividades y las visceralidades.

\section{Interdisciplinaridad/Proceso.}

El proyecto interdisciplinario Feeling the senses empieza en 2016, con un equipo interdisciplinario formado por investigadores que actúan específicamente en el área del arte, del diseño de moda, del diseño de producto, de la arquitectura y de la tecnología, esta unión de los intereses y habilidades en la confección de la pieza amplían las posibilidades proyectuales. Como método de la investigación el equipo realiza reuniones semanales para elaborar la creación, las preguntas, las decisiones y los experimentos, que pueden ser transformadores, reveladores de conocimiento y de verdades (SALLES, 2008).

La propuesta de discusión del significado del corsé se despliega en una búsqueda estructural de un cuerpo extra, que exteriorice con el comportamiento de la pieza vestible - cuando esté en articulación - las subjetividades y revele en la adaptabilidad corpórea del usuario la conexión estimulada con la situación cotidiana.

En este contexto es importante señalar que en un sistema complejo el todo es mayor que la suma de las partes y, en este proyecto, se destaca el aporte positivo del equipo interdisciplinario que formatea lo vestible. El pensamiento complejo, según Morin (2007), busca una perspectiva interdisciplinaria, pero al mismo tiempo, identifica las incertidumbres y lo incompleto de la complejidad.

La virtud sistémica de este proceso se puede situar en lo que Morin (2007) llama de nivel transdisciplinario, en el que se concibe al mismo tiempo, la unidad, en este caso la pieza vestible feeling the senses, según su naturaleza material, como también la complejidad de los fenómenos de asociación y de organización que componen esta pieza. Si la realidad es compleja es necesario que la reflexión sobre ella sea abarcadora y multidimensional, que amplíe las relaciones entre los conceptos que abordan el cuerpo, el movimiento y el vestuario.

Para Morin (2007), la complejidad requiere que su interpretación se realice por medio de relaciones, interrelaciones y fenómenos multidimensionales, configurando un sistema que sugiere la interdisciplinaridad para organizar los agentes y sus relaciones para el desarrollo del proyecto y la obtención de resultados.

Feeling the Senses inicialmente parte de la idea de rigidez y de restricción de movimientos, usando el corsé como una metáfora de las reglas sociales manifestadas en el cuerpo femenino. A través de una interpretación del espacio social histórico y actual como un lugar que restringe las potencialidades de las vidas de las mujeres. Los estudios llevan la construcción de una pieza vestible que evidencie cada movimiento de quien se viste como un enfrentamiento reprendido (Ilustración 1).
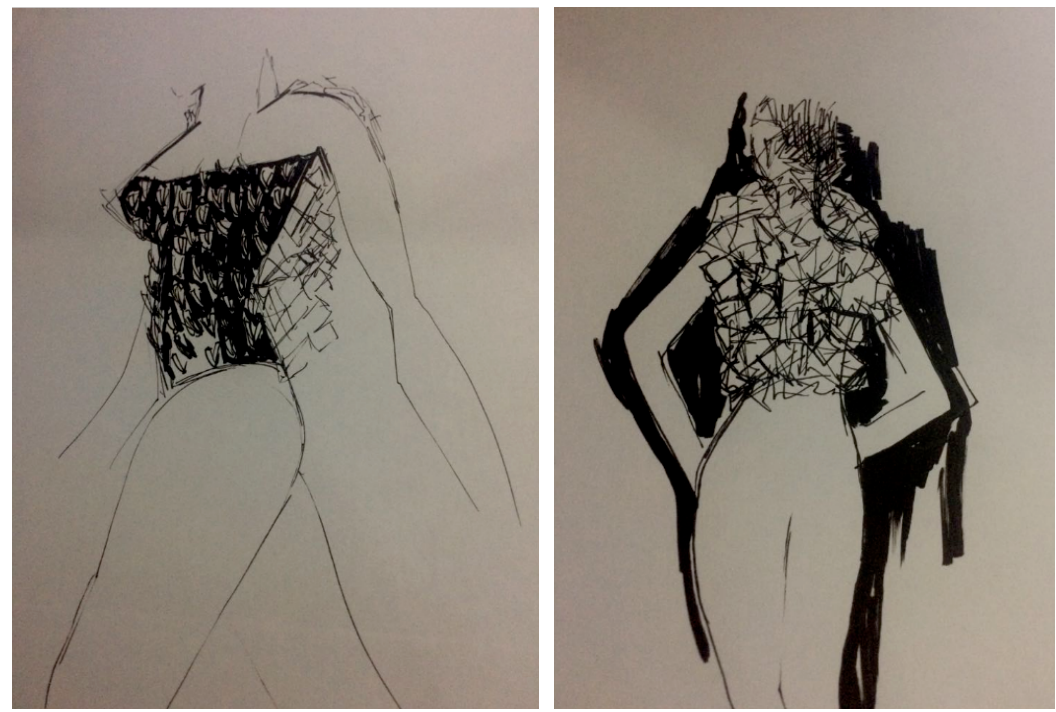

Ilustración 1: Bocetos del proyecto para estudio. 
Gradualmente, el concepto de la pieza pierde el sentido de aprisionamiento y gana la idea de expresión de movimientos, sentidos y sentimientos. El enfoque del equipo se fue dirigiendo a la no linealidad de los códigos sociales de los espacios, y a la inestabilidad de lo cotidiano de las mujeres, con potencialidades que ora favorecen la represión, ora abren brechas para la subversión.

En esta proposición la organización corpórea, el entorno y la materialidad tratan la afectación del cuerpo y del vestirse, y la exposición de sus subjetividades y visceralidades. El diseño busca una estructura que recorre el sentido desde adentro hacia afuera durante la experiencia, la materialización de lo que permanece en lo íntimo del ser y desea transbordar sucede como un grito. "La carne es una especie de escritura viva donde las fuerzas imprimen "vibraciones" y cavan "caminos": el sentido en ella se despliega y en ella se pierde como en un laberinto donde él mismo traza sus propias vías" (LINS, 2002, p.71).

La pieza vestible Felling the Senses en la versión actual confeccionada en malla sigue el modelaje de un cuerpo masculino para transgredir las imposiciones del vestuario femenino. La estructura prevé ataduras en la espalda para la adaptabilidad corporal. La rigidez en la pieza está en los elementos que están bordados directamente en la malla. Realizados en madera según un proyecto digital y corte a láser. En las ilustraciones 2 y 3 , es posible ver los huesos que antes estaban presionados por el corsé, ahora están expuestos. Con la maleabilidad de la malla la pieza se puede modificar por los movimientos de quien la vista, como un corsé que abraza, exterioriza, exagera y potencia pequeños movimientos - en vez de restringirlos. La pieza proyecta para el extra cuerpo lo que viene desde adentro del cuerpo: su visceralidad.
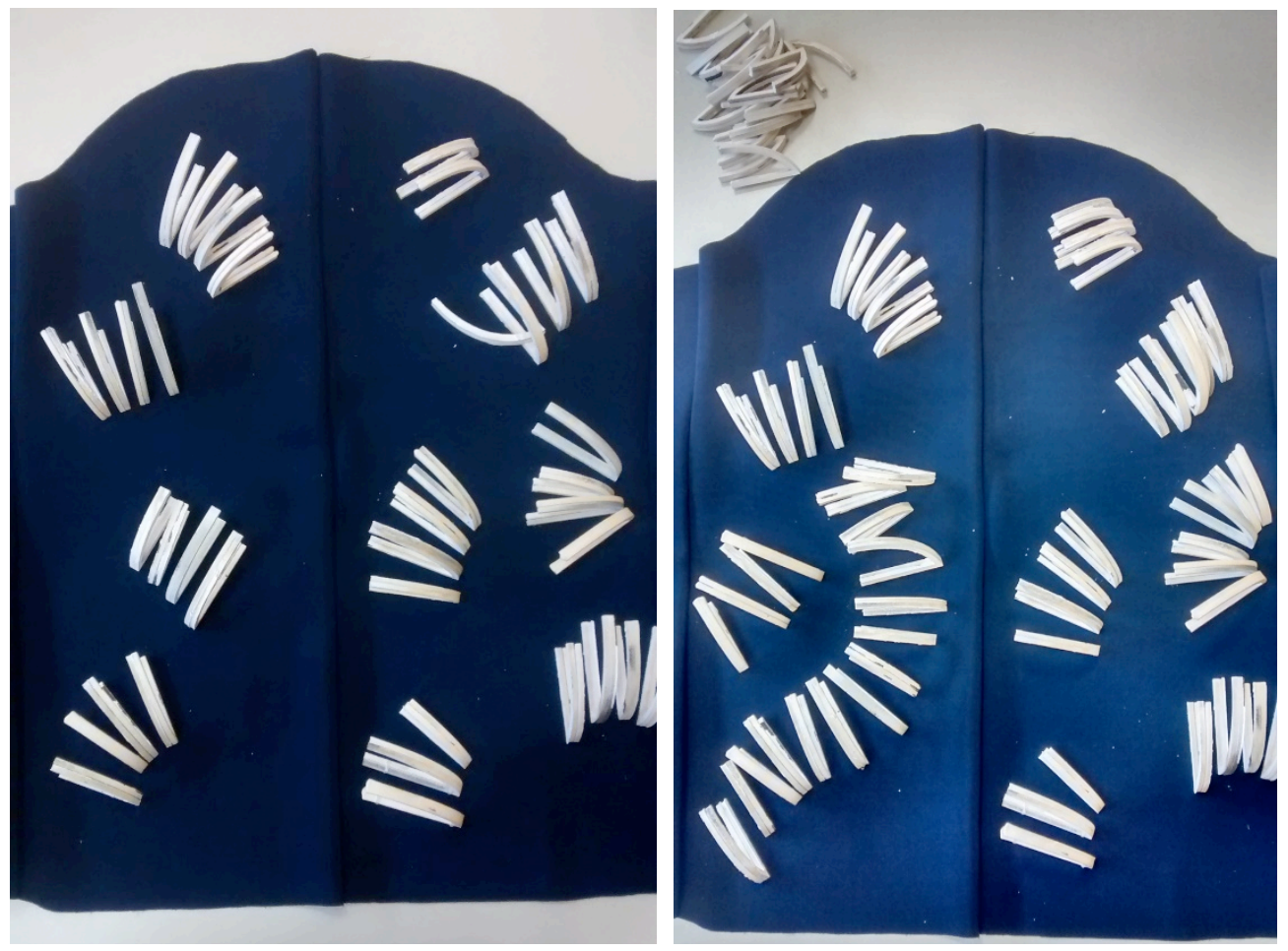

Ilustraciones 2 y 3: Análisis del relleno de los espacios en la pieza vestible.

Otra posibilidad material y procesal, que está en desarrollo para la realización de las piezas que se bordan en la malla externamente, es la impresión 3D, se opta por 3 porcentajes de relleno distintos, que son $30 \%, 50 \%$ y 100\%, para la confección de cada serie de piezas. Cada serie estaba constituida por 3 arcos, que variaban el tamaño y el peso, lo que dejaba a las piezas cada vez más pesadas y densas. Este cambio en el proceso trajo una mayor agilidad y precisión en el desarrollo y en el registro de las informaciones, donde con poco tiempo fue posible crear piezas distintas ampliando las posibilidades. Para el proyecto, todavía en una fase experimental, estamos evaluando la interacción del cuerpo en el hecho de vestir estas piezas con los pesos especificados antes, de acuerdo con su relleno versus espacios vacíos, o sea, en relación a la trama del filamento, donde a medida que aumenta el porcentaje del material disminuye la cantidad de espacios vacíos, lo que deja las piezas más pesadas (ilustración 4). 

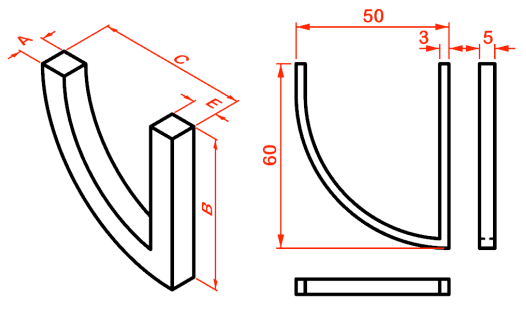

ELEMENTO 1

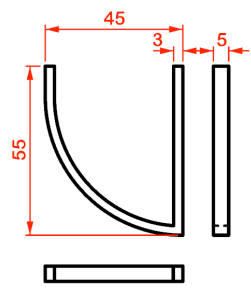

ELEMENTO 2

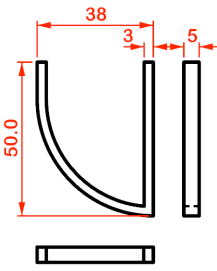

ELEMENTO 3

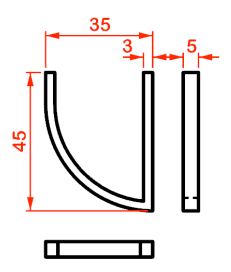

ELEMENTO 4

llustración 4: Diseño de las estructuras producido en Rhino. Marcelo C. Oliveira.

Para la espalda lo vestible presenta un proyecto de pieza desmontable, que se debe acoplar al cuerpo sobre la malla. Inicialmente, se busca un material flexible, que se pueda comprimir y distender. Pero, sólo con la tela lisa, que fue el primer material que se consideró, la visualización del movimiento se mostraba muy sutil junto a un dispositivo mecánico. Entonces, los experimentos pasaron a ser en papel kraft, una estructura con mayor rigidez y más posibilidades de aplicaciones de técnicas no usuales en tela y, que en un futuro puede reemplazarse por una tela con características similares o que pase por un proceso de enrigidecimiento para que se adecue a las funcionalidades propuestas.

La técnica elegida es la dobladura, que transforma el papel de lo plano a lo tridimensional, y conduce a posibilidades de organización y reorganización de su estructura de acuerdo con el estímulo. Así, los movimientos se pueden hacer más evidentes.

Con la dobladura surge la idea del pulmón exteriorizado, pensando en la manifestación de las cuestiones internas y de la fuerza vital, como dobleces que se organizan en módulos interconectados, lo que posibilita la simulación del movimiento de inspiración produciendo la expansión de la estructura y la expiración que causa retracción.

El movimiento de la pieza como principal característica encaminó el experimento a una mayor complejidad en el desarrollo de la forma, pues no contempla simplemente una superficie estática. La superficie considerada en el estudio precisa disponer en su configuración de un sistema que manifieste con énfasis el movimiento propuesto.

El próximo experimento fue un estándar modular de cubos confeccionado en papel continuo sin recortes, que permite el movimiento horizontal, vertical y concomitante. Este sistema, diferente al anterior tiene un límite para la reducción y la expansión, pero con pequeños movimientos es posible percibir su cambio en la forma ${ }^{2}$ (ilustración 5).

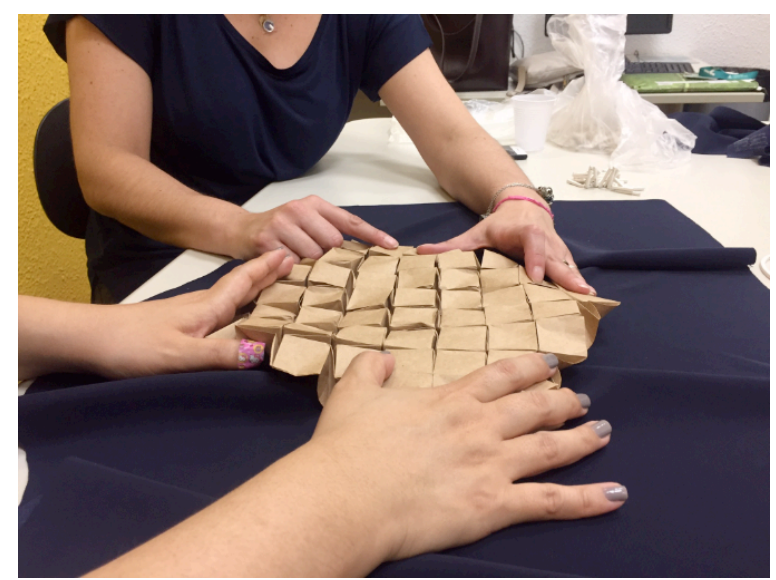

Ilustración 5: Estudio dobladura de papel Kraft como pieza desmontable y con movimientos para la espalda de lo vestible.

Para la inserción del movimiento en lo vestible está en estudio el desarrollo de un mecanismo para accionar las estructuras de frente y de la espalda, que estará posicionado entre la malla y las otras capas del extra cuerpo. Como es necesario realizar movimientos

\footnotetext{
${ }^{2}$ Enlace del movimiento del experiment. Disponible en: <https://www.youtube.com/watch?v=7df-E5nR4Uc>.
} 
distintos, que presenten una reacción a los cuestionamientos señalados anteriormente; se selecciona un sistema de poleas y vástagos articulados. Deberán estar conectados en las extremidades derecha e izquierda de lo vestible, como señala el proyecto.

Esta pieza permite el uso de micro motores que pueden cargarse con pilas AA, lo que facilita el movimiento del usuario durante la experiencia. Con la interconexión de dispositivos computacionales y el uso de placa de arduino que pueden controlar el funcionamiento y la potencia de los motores a partir de los inputs y outputs de la estructura.

\section{Consideraciones Finales}

Esta experiencia es abierta y promueve encuentros en el proceso de creación del proyecto, con varias direcciones y conexiones y así determina una búsqueda y el desvelo de las subjetividades, en este caso, el sentido del cuerpo y un extra cuerpo que interconecta las cuestiones íntimas durante el movimiento en la cotidianeidad.

Después del diálogo con el corsé, el proyecto revela otra posibilidad de interacción con el espacio a través de una interfaz vestible, que manifiesta la condición del usuario, en medio de una trama compleja de elementos y agentes, cuando están articulados. Se cuestionan posibles reacciones con la realización de una materialidad que trata el hecho de vestirse y sus experiencias, con una estructura que busca posibles brechas entre las subjetividades del sentir y del vivir.

Esta búsqueda proyectual y conceptual vislumbra la continuidad de los experimentos y los cuestionamientos en los que el significado del cuerpo y del vestuario se potencian con la estructura y con la inserción de la tecnología que puede ampliar los aspectos sensoriales y cognitivos (ilustraciones 6 y 7 ).
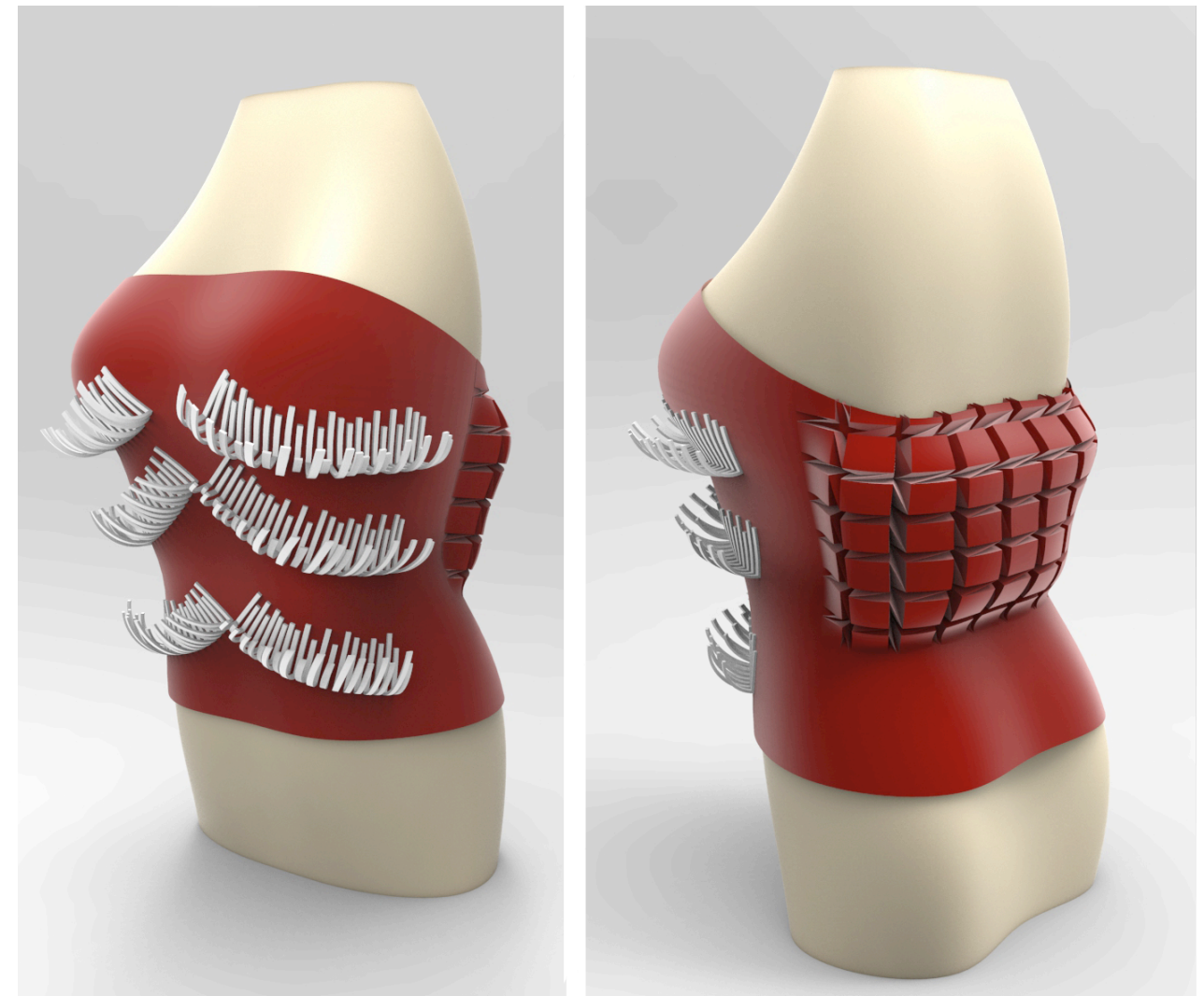

Ilustraciones 6 y 7: Desarrollo del proyecto en Rhino, de lo vestible Feeling the senses (frente y dorso). Marcelo C. Oliveira. 
Carvalho, Agda; Trovo, Priscila; Kishimoto, Grace; Rosa, Natalia; Kulaif, Patricia; Valli, Adriana; Braga, Rechilene Maia; Da Hora, Miguel Proyecto interdisciplinar Feeling the senses: El vestir como experiencia

III CONGRESO INTERNACIONAL DE INVESTIGACIÓN EN ARTES VISUALES :: ANIAV 2017 :: GLOCAL [codificar, mediar, transformar, vivir] http://dx.doi.org/10.4995/ANIAV.2017.4941

\section{FUENTES REFERENCIALES.}

DORFMAN. B. Arquitetura e Representação: as Casas de Papel, de Peter Eisenman e textos da desconstrução, de Jacques Derrida, años 60 a 80. Universidad Federal do Rio Grande do Sul: 2009.

LINS, Daniel; GADELHA, Sylvio (Org.). Nietzsche e Deleuze: que pode o corpo. Rio de Janeiro: Relume, 2002

MASSEY, Dorren. Pelo Espaço: Uma nova política da espacialidade. Rio de Janeiro: Bertrand Brasil, 2008.

MORIN, Edgar. Introdução ao pensamento complexo. Porto Alegre: Sulina, 2007.

ENTWISTLE, J. The Fashioned Body: Fashion, dress and Modern Social Theory. Maiden: Polity Press, 2000.

SALLES, Cecília Almeida. Redes da Criação. Construção da obra de arte. São Paulo: Horizonte, 2008.

STEELE, Valerie. The corset: a cultural history. New Haven: Yale University Press, 2011.

VARELA, Francisco. O reencantamento do concreto. Cadernos de subjetividade, v. 1, n. 1, p. 72-86, 2003. 